\title{
A Characterization of Superlinear Convergence and Its Application to Quasi-Newton Methods*
}

\author{
By J. E. Dennis, Jr. and Jorge J. Moré
}

\begin{abstract}
Let $F$ be a mapping from real $n$-dimensional Euclidean space into itself. Most practical algorithms for finding a zero of $F$ are of the form

$$
x_{k+1}=x_{k}-B_{k}^{-1} F x_{k},
$$

where $\left\{B_{k}\right\}$ is a sequence of nonsingular matrices. The main result of this paper is a characterization theorem for the superlinear convergence to a zero of $F$ of sequences of the above form. This result is then used to give a unified treatment of the results on the superlinear convergence of the Davidon-Fletcher-Powell method obtained by Powell for the case in which exact line searches are used, and by Broyden, Dennis, and More for the case without line searches. As a by-product, several results on the asymptotic behavior of the sequence $\left\{B_{k}\right\}$ are obtained.

An interesting aspect of these results is that superlinear convergence is obtained without any consistency conditions; i.e., without requiring that the sequence $\left\{B_{k}\right\}$ converge to the Jacobian matrix of $F$ at the zero. In fact, a modification of an example due to Powell shows that most of the known quasi-Newton methods are not, in general, consistent. Finally, it is pointed out that the above-mentioned characterization theorem applies to other single and double rank quasi-Newton methods, and that the results of this paper can be used to obtain their superlinear convergence.
\end{abstract}

1. Introduction. This paper will be concerned with iterations of the form

$$
x_{k+1}=x_{k}-B_{k}^{-1} F x_{k}, \quad k=0,1, \cdots,
$$

for the solution of $F x=0$, where $F: R^{n} \rightarrow R^{n}$ is a mapping of Euclidean $n$-space $R^{n}$ into itself. Each nonsingular matrix $B_{k}$ is directly or indirectly (as in various quasiNewton methods) intended to approximate $F^{\prime}\left(x_{k}\right)$, the Jacobian matrix of $F$ at $x_{k}$. Virtually all practical methods for generating a sequence $\left\{x_{k}\right\}$ of approximate zeros of $F$ have the basic form (1.1) although they are often implemented in their damped version,

$$
x_{k+1}=x_{k}-\lambda_{k} B_{k}^{-1} F x_{k}, \quad k=0,1, \cdots,
$$

where $\lambda_{k}$ is a scalar chosen to prevent divergence of the sequence $\left\{x_{k}\right\}$. Even in this case, the hope is always that $\lambda_{k}=1$ will suffice near $x^{*}$, a zero of $F$.

No matter which of (1.1) or (1.2) is used to generate $\left\{x_{k}\right\}$, the utility of the method

Received March 12, 1973.

AMS (MOS) subject classifications (1970). Primary 65H10, 65K05.

Key words and phrases. Quasi-Newton methods, variable metric methods, superlinear convergence, iterative methods for nonlinear equations.

* This research was supported in part by the National Science Foundation under Grant GJ27528.

Copyright @ 1974, American Mathematical Society 
is in large part dependent on the speed with which $\left\{x_{k}\right\}$ converges to $x^{*}$. In order to be practical, a method should be at least $Q$-linear (for material on rates of convergence see [7, Chapter 9]) when $F$ satisfies reasonable hypotheses and $\left\{x_{k}\right\}$ converges to $x^{*}$; i.e., for some $r \in(0,1),\left\|x_{k+1}-x^{*}\right\| \leqq r\left\|x_{k}-x^{*}\right\|, k=0,1, \cdots$. Furthermore, if the method is to be competitive then it should in fact be $Q$-superlinear, i.e., $\left\{\left\|x_{k+1}-x^{*}\right\| /\left\|x_{k}-x^{*}\right\|\right\}$ converges to zero with $k$. Higher orders of convergence are desirable when obtainable without unreasonable additional computations although for general problems, orders higher than two are of minimal importance.

The standard technique for proving that a method is $Q$-superlinear has always been to show that as $\left\{x_{k}\right\}$ converges to $x^{*},\left\{B_{k}\right\}$ converges to $F^{\prime}\left(x^{*}\right)$. It is well known that this consistency condition is sufficient but not necessary. (See Voigt [11] for a compendium of interesting theorems and examples.) Since, until recently, all the practical methods for which there were published proofs of $Q$-superlinearity satisfied this property, it seemed to be effectively necessary.

Recently, M. J. D. Powell [8] proved that the Davidon-Fletcher-Powell method is $Q$-superlinear when implemented in the form (1.2) with $\lambda_{k}$ chosen by the standard line minimization criterion. On the other hand, Broyden, Dennis and Moré [2] showed that many of the most important quasi-Newton methods are $Q$-superlinear when implemented in the form (1.1). Powell [9] has also furnished an example which shows that the celebrated Davidon-Fletcher-Powell method is indeed a practical $Q$-superlinear method which does not have the property that $\left\{B_{k}\right\}$ converges to $F^{\prime}\left(x^{*}\right)$. When properly modified, this example shows that most of the known quasiNewton methods are not, in general, consistent. In this paper, a quasi-Newton method refers to an algorithm of the form (1.1) or (1.2) where $B_{k+1}$ is obtained by adding a matrix of rank at most two to $B_{k}$. For example, all the algorithms in the Huang [5] class are quasi-Newton methods, but the Goldstein-Price [4] algorithm is not a quasi-Newton method.

Although the Powell and Broyden, Dennis, Moré convergence proofs are quite distinct, the parts of both papers which deal with $Q$-superlinearity turn out to be based on the same principle. The main purpose of this paper is to enunciate that principle and some of its consequences.

In the next section, we prove a characterization theorem for $Q$-superlinear convergence to a zero of $F$ which applies to sequences generated by either (1.1) or (1.2). We also obtain a simple, but apparently new, justification for using $\left\|x_{k+1}-x_{k}\right\|$ as an estimate for $\left\|x_{k}-x^{*}\right\|$.

In Section 3, we apply our results to the Davidon-Fletcher-Powell method and show how the superlinear results of Powell and those of Broyden, Dennis and Moré can be obtained, while in Section 4 we indicate how these results can be applied to other quasi-Newton methods and we give Powell's example. We conclude with some general remarks. In particular, we compare our results to those obtained by McCormick and Ritter [6], and Ritter [10].

2. Characterization of Superlinear Convergence. Let $\|\cdot\|$ denote an arbitrary vector norm in $R^{n}$ or the operator norm it induces in $L\left(R^{n}\right)$-the space of real matrices of order $n$. Now recall [7, Chapter 9] that if $\left\{x_{k}\right\} \subset R^{n}$ converges to $x^{*}$, then $\left\{x_{k}\right\}$ converges $Q$-superlinearly to $x^{*}$ if and only if either $x_{k}=x^{*}$ for all sufficiently large $k$ or $x_{k} \neq x^{*}$ for $k \geqq k_{0}$ and 


$$
\lim _{k \rightarrow+\infty}\left\|x_{k+1}-x^{*}\right\| /\left\|x_{k}-x^{*}\right\|=0 .
$$

In this paper, when we assume that $\left\{x_{k}\right\}$ converges to $x^{*}$, we will also make the unstated assumption that $x_{k} \neq x^{*}$ for all sufficiently large $k$. In the context of (1.1), this simply means $x_{k+1} \neq x_{k}$ for any $k \geqq 0$ and is thus quite reasonable.

With these preliminaries out of the way, it is possible to proceed with the stated purpose of this section, the development of a characterization theorem for methods of the form (1.1) which are $Q$-superlinearly convergent. We begin with a result that gives a necessary condition for an arbitrary sequence.

LeMma 2.1. Let $\left\{x_{k}\right\} \subset R^{n}$ converge $Q$-superlinearly to $x^{*}$. Then

$$
\lim _{k \rightarrow+\infty}\left\|x_{k+1}-x_{k}\right\| /\left\|x_{k}-x^{*}\right\|=1 .
$$

Proof. Just note that

$$
\left|\frac{\left\|x_{k+1}-x_{k}\right\|}{\left\|x_{k}-x^{*}\right\|}-\frac{\left\|x_{k}-x^{*}\right\|}{\left\|x_{k}-x^{*}\right\|}\right| \leqq \frac{\left\|x_{k+1}-x^{*}\right\|}{\left\|x_{k}-x^{*}\right\|} .
$$

The converse does not hold. For example, if $x_{2 k-1}=(k !)^{-1}$ and $x_{2 k}=2 x_{2 k-1}$ for $k \geqq 1$, then this sequence satisfies (2.1) with $x^{*}=0$, but does not converge $Q$ superlinearly to zero.

Lemma 2.1 justifies the very commonly used computational technique of estimating $\left\|x_{k}-x^{*}\right\|$ with $\left\|x_{k+1}-x_{k}\right\|$, if the underlying method is $Q$-superlinear.

To obtain a characterization of $Q$-superlinear convergence for (1.1), we will assume that $F$ is defined and (Gateaux) differentiable in an open, convex set $D$; that is, its Jacobian matrix $F^{\prime}(x)$ satisfies

$$
\lim _{t \rightarrow 0} \frac{F(x+t h)-F(x)}{t}=F^{\prime}(x) h
$$

for each $x$ in $D$ and $h$ in $R^{n}$. See, for example, [7, Chapter 3]. This facilitates the proofs but the existence of $F^{\prime}(x)$ is all that is really needed.

THEOREM 2.2. Let $F: R^{n} \rightarrow R^{n}$ be differentiable in the open, convex set $D$ in $R^{n}$, and assume that for some $x^{*}$ in $D F^{\prime}$ is continuous at $x^{*}$ and $F^{\prime}\left(x^{*}\right)$ is nonsingular. Let $\left\{B_{k}\right\}$ in $L\left(R^{n}\right)$ be a sequence of nonsingular matrices and suppose that for some $x_{0}$ in $D$ the sequence $\left\{x_{k}\right\}$ where

$$
x_{k+1}=x_{k}-B_{k}^{-1} F x_{k}
$$

remains in $D$ and converges to $x^{*}$. Then $\left\{x_{k}\right\}$ converges $Q$-superlinearly to $x^{*}$ and $F x^{*}=0$ if and only if

$$
\lim _{k \rightarrow+\infty} \frac{\left\|\left[B_{k}-F^{\prime}\left(x^{*}\right)\right]\left(x_{k+1}-x_{k}\right)\right\|}{\left\|x_{k+1}-x_{k}\right\|}=0 .
$$

Proof. Assume first that (2.3) holds. Since

$$
\begin{aligned}
{\left[B_{k}-F^{\prime}\left(x^{*}\right)\right]\left(x_{k+1}-x_{k}\right) } & =-F x_{k}-F^{\prime}\left(x^{*}\right)\left(x_{k+1}-x_{k}\right) \\
& =F x_{k+1}-F x_{k}-F^{\prime}\left(x^{*}\right)\left(x_{k+1}-x_{k}\right)-F x_{k+1},
\end{aligned}
$$

the continuity of $F^{\prime}$ at $x^{*}$ and (2.3) imply that

$$
\lim _{k \rightarrow+\infty}\left\|F x_{k+1}\right\| /\left\|x_{k+1}-x_{k}\right\|=0 .
$$


Hence, $F x^{*}=0$, and since $F^{\prime}\left(x^{*}\right)$ is nonsingular, there is a $\beta>0$ such that

$$
\left\|F x_{k+1}\right\|=\left\|F x_{k+1}-F x^{*}\right\| \geqq \beta\left\|x_{k+1}-x^{*}\right\|,
$$

and, therefore,

$$
\frac{\left\|F x_{k+1}\right\|}{\left\|x_{k+1}-x_{k}\right\|} \geqq \frac{\beta\left\|x_{k+1}-x^{*}\right\|}{\left\|x_{k+1}-x^{*}\right\|+\left\|x_{k}-x^{*}\right\|}=\beta \frac{\rho_{k}}{1+\rho_{k}}
$$

where $\rho_{k}=\left\|x_{k+1}-x^{*}\right\| /\left\|x_{k}-x^{*}\right\|$. Thus, (2.5) implies that $\rho_{k} /\left(1+\rho_{k}\right)$ converges to zero and hence $\left\{\rho_{k}\right\}$ also converges to zero as desired.

Conversely, assume that $\left\{x_{k}\right\}$ converges $Q$-superlinearly to $x^{*}$ and $F x^{*}=0$. Since

$$
\frac{\left\|F x_{k+1}\right\|}{\left\|x_{k+1}-x_{k}\right\|}=\frac{\left\|F x_{k+1}-F x^{*}\right\|}{\left\|x_{k}-x^{*}\right\|} \cdot \frac{\left\|x_{k}-x^{*}\right\|}{\left\|x_{k+1}-x_{k}\right\|},
$$

Lemma 2.1 and the hypotheses on $F^{\prime}$ imply that (2.5) holds. It then follows from (2.4) that condition (2.3) is satisfied.

In some cases, it is very easy to verify that (2.3) holds. For example, in a Newton or discretized Newton method, $\left\{B_{k}\right\}$ converges to $F^{\prime}\left(x^{*}\right)$ and hence $(2.3)$ holds. We will later show that (2.3) also holds for many quasi-Newton methods, even though in this case $\left\{B_{k}\right\}$ does not necessarily converge to $F^{\prime}\left(x^{*}\right)$ (see Section 4 ).

The Newton, discretized Newton, and quasi-Newton methods are sometimes implemented in the form (1.2) where the sequence $\left\{\lambda_{k}\right\}$ is chosen so as to enlarge the domain of convergence. The next result shows that this sequence will still be $Q$-superlinear if and only if $\left\{\lambda_{k}\right\}$ converges to unity.

COROLlARY 2.3. Let $F: R^{n} \rightarrow R^{n}$ satisfy the hypotheses of Theorem 2.2 on the set $D$. Let $\left\{B_{k}\right\}$ be a sequence of nonsingular matrices, and suppose that for some $x_{0}$ in $D$ the sequence $\left\{x_{k}\right\}$ generated by (1.2) remains in $D$ and converges to $x^{*}$. If (2.3) holds, then $\left\{x_{k}\right\}$ converges $Q$-superlinearly to $x^{*}$ where $F x^{*}=0$ if and only if $\left\{\lambda_{k}\right\}$ converges to unity.

Proof. Assume that $\left\{x_{k}\right\}$ converges $Q$-superlinearly to $x^{*}$ and $F x^{*}=0$. By Theorem 2.2, we must have

$$
\lim _{k \rightarrow+\infty} \frac{\left\|\left[\lambda_{k}^{-1} B_{k}-F^{\prime}\left(x^{*}\right)\right]\left(x_{k+1}-x_{k}\right)\right\|}{\left\|x_{k+1}-x_{k}\right\|}=0,
$$

and therefore (2.3) implies that

$$
\lim _{k \rightarrow+\infty}\left\|\left(\lambda_{k}^{-1}-1\right) B_{k}\left(x_{k+1}-x_{k}\right)\right\| /\left\|x_{k+1}-x_{k}\right\|=0 .
$$

But $B_{k}\left(x_{k+1}-x_{k}\right)=-\lambda_{k} F x_{k}$, so that

$$
\lim _{k \rightarrow+\infty}\left\|\left(\lambda_{k}-1\right) F x_{k}\right\| /\left\|x_{k+1}-x_{k}\right\|=0 \text {. }
$$

Now $F^{\prime}\left(x^{*}\right)$ is nonsingular, and thus there is a $\beta>0$ such that $\left\|F x_{k}\right\| \geqq \beta\left\|x_{k}-x^{*}\right\|$. Therefore, Lemma 2.1 implies that $\left\{\lambda_{k}\right\}$ must converge to unity.

Conversely, if $\left\{\lambda_{k}\right\}$ converges to unity, it is clear from (2.3) that (2.6) holds. Hence, Theorem 2.2 guarantees that the sequence given by (1.2) converges $Q$-superlinearly to $x^{*}$ where $F x^{*}=0$.

If (2.3) holds, Corollary 2.3 explains why techniques for finding $\left\{\lambda_{k}\right\}$ must even- 
tually produce values close to unity if there is to be $Q$-superlinear convergence to a zero of $F$. For example, in the often-quoted technique of Goldstein and Price [4] for discretized Newton methods, $\lambda_{k}=1$ for all large $k$. Corollary 2.3 then explains why they were able to obtain $Q$-superlinear convergence. Later on, we will prove that a number of quasi-Newton methods also satisfy (2.3) so that the above remarks also apply to these methods.

Finally, note that the results of this section can be used to prove that certain methods of the form (2.2) are not $Q$-superlinearly convergent to a zero of $F$. For example, in the "reset" methods, one sets $\boldsymbol{B}_{k_{i}}=\boldsymbol{B}$ for some fixed nonsingular matrix, and some subsequence $\left\{k_{i}\right\}$. If $\left\{x_{k}\right\}$ converges $Q$-superlinearly to a zero of $F$, then Theorem 2.2 implies that (2.3) holds, and thus $B s=F^{\prime}\left(x^{*}\right) s$ for some vector $s$ with $\|s\|=1$. In particular, 1 is an eigenvalue of $B^{-1} F^{\prime}\left(x^{*}\right)$. This indicates that unless $B$ is chosen with great care, resetting will prevent $Q$-superlinear convergence to a zero of $F$.

3. Superlinear Convergence of the Davidon-Fletcher-Powell Method. For a mapping $F: R^{n} \rightarrow R^{n}$, the Davidon-Fletcher-Powell method is defined by

$$
x_{k+1}=x_{k}-\lambda_{k} H_{k} F x_{k}
$$

where

$$
H_{k+1}=H_{k}+s_{k} s_{k}^{T} / s_{k}^{T} y_{k}-H_{k} y_{k} y_{k}^{T} H_{k} / y_{k}^{T} H_{k} y_{k},
$$

and

$$
s_{k}=x_{k+1}-x_{k}, \quad y_{k}=F x_{k+1}-F x_{k} .
$$

There are two results which guarantee that the Davidon-Fletcher-Powell method is $Q$-superlinearly convergent. The first result is due to Powell [8]; he assumes that (a) $F$ is the gradient of a uniformly convex functional $f: R^{n} \rightarrow R^{1}$, (b) $\lambda_{k}$ is chosen so that

$$
f\left(x_{k}-\lambda_{k} p_{k}\right)=\min \left\{f\left(x_{k}-\lambda p_{k}\right): \lambda \geqq 0\right\}
$$

where $p_{k}=H_{k} \nabla f\left(x_{k}\right)$, and (c) $F$ is a continuously differentiable mapping on $\boldsymbol{R}^{n}$ which satisfies the one-sided Lipschitz condition

$$
\left\|F^{\prime}(x)-F^{\prime}\left(x^{*}\right)\right\| \leqq L\left\|x-x^{*}\right\|
$$

in the level set $\left\{x: f(x) \leqq f\left(x_{0}\right)\right\}$. Here, $x^{*}$ is the unique solution of $F x=0$. Under these hypotheses, Powell proved that for any $x_{0}$ in $R^{n}$ and any symmetric, positive definite $H_{0}$, the Davidon-Fletcher-Powell method is $Q$-superlinearly convergent to $x^{*}$.

On the other hand, Broyden, Dennis, and Moré [2] assumed that (a) $F: R^{n} \rightarrow R^{n}$ is differentiable in an open convex neighborhood $D$ of a point $x^{*}$ for which $F x^{*}=0$ and $F^{\prime}\left(x^{*}\right)$ is symmetric and positive definite, and that (b) $F^{\prime}$ satisfies the one-sided Lipschitz condition

$$
\left\|F^{\prime}(x)-F^{\prime}\left(x^{*}\right)\right\| \leqq K\left\|x-x^{*}\right\|^{p}
$$

for some $p>0$ and all $x$ in $D$. With these hypotheses, they proved that if $\left(x_{0}, H_{0}\right)$ is sufficiently close to $\left(x^{*}, F^{\prime}\left(x^{*}\right)^{-1}\right)$, then the Davidon-Fletcher-Powell method with $\lambda_{k}=1$ for all $k$ is $Q$-superlinearly convergent to $x^{*}$. 
These two convergence results are really more complementary than comparable and their proofs are quite distinct. We will show now that the results of Section 2 not only give a unified treatment of both rate of convergence proofs, but lead to the conclusion that Powell's minimizing $\left\{\lambda_{k}\right\}$ converges to 1 .

It is well known that if $H_{k+1}$ is defined from $H_{k}, s_{k}$ and $y_{k}$ by (3.2) and if $H_{k}^{-1}=B_{k}$ exists, then, as long as $y_{k}{ }^{T} s_{k} \neq 0, H_{k+1}{ }^{-1}$ is defined by

$$
\begin{aligned}
B_{k+1}= & B_{k}+\frac{\left(y_{k}-B_{k} s_{k}\right) y_{k}^{T}+y_{k}\left(y_{k}-B_{k} s_{k}\right)^{T}}{y_{k}^{T} s_{k}} \\
& -\frac{s_{k}^{T}\left(y_{k}-B_{k} s_{k}\right) y_{k} y_{k}^{T}}{\left(y_{k}^{T} s_{k}\right)^{2}}
\end{aligned}
$$

Note that $\left\{B_{k}\right\}$ is defined provided the sequences $\left\{y_{k}\right\}$ and $\left\{s_{k}\right\}$ satisfy $y_{k}{ }^{T} s_{k} \neq 0$. We assume that $\left\{y_{k}\right\}$ and $\left\{s_{k}\right\}$ are generated by (3.3), but, for the moment, we will leave $\left\{x_{k}\right\}$ unspecified.

We now plan to investigate the behavior of the sequence $\left\{B_{k}\right\}$ generated by (3.5). For this, first recall that for $A \in L\left(R^{n}\right)$ the Frobenius norm is defined by

$$
\|A\|_{F}^{2}=\sum_{i, j=1}^{n}\left|a_{i j}\right|^{2} \text {. }
$$

We also need the following special case of a result of Broyden, Dennis, and Moré [2].

LEMMA 3.1. Let $M \in L\left(R^{n}\right)$ be a nonsingular symmetric matrix such that

$$
\left\|M y-M^{-1} s\right\| \leqq \beta\left\|M^{-1} s\right\|
$$

for some $\beta \in\left[0, \frac{1}{3}\right]$ and vectors $y$ and $s$ in $R^{n}$ with $s \neq 0$. Then $y^{T} s>0$ and thus $\bar{B} \in$ $L\left(R^{n}\right)$ can be defined by

$$
\bar{B}=B+\frac{(y-B s) y^{T}+y(y-B s)^{T}}{y^{T} s}-\frac{s^{T}(y-B s) y y^{T}}{\left(y^{T} s\right)^{2}}
$$

where $B \in L\left(R^{n}\right)$ is symmetric. Moreover, if $\|\cdot\|_{M}$ is the matrix norm defined by

$$
\|Q\|_{M}=\|M Q M\|_{F} \text {, }
$$

then there are positive constants $\alpha, \alpha_{1}$ and $\alpha_{2}$ (depending only on $M$ and $n$ ) such that, for any symmetric $A \in L\left(R^{n}\right)$,

$$
\begin{aligned}
\|\bar{B}-A\|_{M} \leqq & {\left[\left(1-\alpha \theta^{2}\right)^{1 / 2}+\alpha_{1}\left\|M y-M^{-1} s\right\| /\left\|M^{-1} s\right\|\right]\|B-A\|_{M} } \\
& +\alpha_{2}\|y-A s\| /\left\|M^{-1} s\right\|
\end{aligned}
$$

where $\alpha \in(0,1]$, and

$$
\theta=\|M[B-A] s\| /\left(\|B-A\|_{M}\left\|M^{-1} s\right\|\right), \text { for } B \neq A,
$$

with $\theta=0$ otherwise.

In [2] a more general result was given, but only for the case when $\|\cdot\|$ was the $l_{2}$ norm and $B \neq A$. Those restrictions are unnecessary in the present case. Note that this lemma needs only a way of choosing $M$ in order to be applicable to $\left\{B_{k}\right\}$. In the following lemma, we show that if $F^{\prime}\left(x^{*}\right)$ is symmetric and positive definite, then $M$ can be taken to be the symmetric, positive definite, square root of $F^{\prime}\left(x^{*}\right)^{-1}$, i.e., $F^{\prime}\left(x^{*}\right)^{-1 / 2}$. It is interesting to note that we do not assume $\left\{x_{k}\right\}$ is generated by (3.1) or that $\left\{H_{k}\right\}$ exists or even that $F\left(x^{*}\right)=0$. 
LEMMA 3.2. Let $F: R^{n} \rightarrow R^{n}$ be differentiable in an open, convex neighborhood $D$ of a point $x^{*}$ for which $F^{\prime}\left(x^{*}\right)$ is symmetric and positive definite, and suppose that $F$ satisfies the Lipschitz condition (3.4) in a neighborhood of $x^{*}$. If for some sequence $\left\{x_{k}\right\} \subset D$ which converges to $x^{*}$, we define $\left\{s_{k}\right\}$ and $\left\{y_{k}\right\}$ by (3.3), then $y_{k}{ }^{T} s_{k}>0$ for $k \geqq k_{0}$. Moreover, for any symmetric $B_{k_{0}} \in L\left(R^{n}\right)$ the sequence $\left\{B_{k}\right\}$ is well defined by (3.5) for $k \geqq k_{0}$, and there are positive constants $\alpha, \alpha_{3}$, and $\alpha_{4}$ such that

$$
\left\|B_{k+1}-F^{\prime}\left(x^{*}\right)\right\|_{M} \leqq\left[\left(1-\alpha \theta_{k}^{2}\right)^{1 / 2}+\alpha_{3} \sigma_{k}\right]\left\|B_{k}-F^{\prime}\left(x^{*}\right)\right\|_{M}+\alpha_{4} \sigma_{k},
$$

where $\sigma_{k}=\max \left\{\left\|x_{k+1}-x^{*}\right\|^{p},\left\|x_{k}-x^{*}\right\|^{p}\right\}$. Here $\alpha \in(0,1],\|\cdot\|_{M}$ is defined by (3.7) with $M=F^{\prime}\left(x^{*}\right)^{-1 / 2}$, and

$$
\theta_{k}=\frac{\left\|M\left[B_{k}-F^{\prime}\left(x^{*}\right)\right] s_{k}\right\|}{\left\|B_{k}-F^{\prime}\left(x^{*}\right)\right\|_{M}\left\|M^{-1} s_{k}\right\|}, \quad \text { for } B_{k} \neq F^{\prime}\left(x^{*}\right),
$$

and $\theta_{k}=0$ otherwise.

Proof. For $M=F^{\prime}\left(x^{*}\right)^{-1 / 2}$, we have $\left\|M y_{k}-M^{-1} s_{k}\right\| \leqq\|M\|\left\|y_{k}-F^{\prime}\left(x^{*}\right) s_{k}\right\|$, and it is not difficult (e.g. Lemma 3.1 in [2]) to verify that if (3.4) holds then

$$
\left\|y_{k}-F^{\prime}\left(x^{*}\right) s_{k}\right\| \leqq K \max \left\{\left\|x_{k+1}-x^{*}\right\|^{p},\left\|x_{k}-x^{*}\right\|^{p}\right\}\left\|s_{k}\right\| .
$$

Hence, (3.6) is satisfied if $k_{0}$ is taken so that $K\|M\|\left\|x_{k}-x^{*}\right\|^{p} \leqq \frac{1}{3}$ for $k \geqq k_{0}$. The result now follows from Lemma 3.1.

Our next task is to deduce properties of $\left\{B_{k}\right\}$ from (3.8). The following result is applicable if we assume that

$$
\sum_{k=1}^{\infty}\left\|x_{k}-x^{*}\right\|^{p}<+\infty .
$$

LEMMA 3.3. Let $\left\{\phi_{k}\right\}$ and $\left\{\delta_{k}\right\}$ be sequences of nonnegative numbers such that

$$
\phi_{k+1} \leqq\left(1+\delta_{k}\right) \phi_{k}+\delta_{k}
$$

and

$$
\sum_{k=1}^{\infty} \delta_{k}<+\infty
$$

Then $\left\{\phi_{k}\right\}$ converges.

Proof. We first show that $\left\{\phi_{k}\right\}$ is bounded (above). Let

$$
\mu_{k}=\prod_{i=1}^{k-1}\left(1+\delta_{i}\right) .
$$

Then $\mu_{k} \geqq 1$, and (3.11) implies that $\mu_{k} \leqq \mu$ for some constant $\mu$. But (3.10) yields

$$
\phi_{k+1} / \mu_{k+1} \leqq \phi_{k} / \mu_{k}+\delta_{k} / \mu_{k+1} \leqq \phi_{k} / \mu_{k}+\delta_{k},
$$

and therefore

$$
\phi_{m+1} / \mu_{m+1} \leqq \phi_{1} / \mu_{1}+\sum_{k=1}^{m} \delta_{k},
$$

which in view of (3.11) and the boundedness of $\left\{\mu_{k}\right\}$ implies that $\left\{\phi_{k}\right\}$ is bounded.

Since $\left\{\phi_{k}\right\}$ is bounded, it certainly has at least one limit point. Suppose there are subsequences $\left\{\phi_{k_{n}}\right\},\left\{\phi_{k_{m}}\right\}$ which converge respectively to limit points $\phi^{\prime}$ and $\phi^{\prime \prime}$. 
We will show that $\phi^{\prime} \leqq \phi^{\prime \prime}$ and by symmetry $\phi^{\prime \prime} \leqq \phi^{\prime}$. Thus $\phi^{\prime \prime}=\phi^{\prime}$ and consequently $\left\{\phi_{k}\right\}$ converges.

Let $\phi$ bound $\left\{\phi_{k}\right\}$. It follows from (3.10) that if $k_{n} \geqq k_{m}$ then $\phi_{k_{n}}-\phi_{k_{m}} \leqq$ $(1+\phi) \sum_{i=k_{m}}^{\infty} \delta_{j}$. Thus, by the choice of $\left\{k_{n}\right\}$,

$$
\phi^{\prime}-\phi_{k_{m}} \leqq(1+\phi) \sum_{i=k_{m}}^{\infty} \delta_{i}
$$

and by the choice of $\left\{k_{m}\right\}, \phi^{\prime}-\phi^{\prime \prime} \leqq 0$. This completes the proof.

THEOREM 3.4. Assume that $F: R^{n} \rightarrow R^{n}$ satisfies the hypotheses of Lemma 3.2 on the set $D$ and let $\left\{x_{k}\right\}$ be a sequence in $D$ which satisfies (3.9). Then there is a positive integer $k_{0}$ such that for any symmetric $B_{k_{0}} \in L\left(R^{n}\right)$ the sequences $\left\{s_{k}\right\},\left\{y_{k}\right\}$ and $\left\{B_{k}\right\}$ are well defined for $k \geqq k_{0}$ by (3.3) and (3.5). Furthermore,

(i) $\lim _{k \rightarrow \infty}\left\|F^{\prime}\left(x^{*}\right)^{-1 / 2} B_{k} F^{\prime}\left(x^{*}\right)^{-1 / 2}-I\right\|_{F}$ exists, and

(ii) $\lim _{k \rightarrow \infty}\left(\|\left[B_{k}-F^{\prime}\left(x^{*}\right)\right] s_{k}|| /|| s_{k}||\right)=0$.

Proof. Statement (i) follows directly from Lemmas 3.2 and 3.3. To prove statement (ii), note that since $\left(1-\alpha \theta_{k}{ }^{2}\right)^{1 / 2} \leqq 1-(\alpha / 2) \theta_{k}{ }^{2}$, Eq. (3.8) can be written as

$$
\begin{aligned}
\left(\alpha \theta_{k}^{2} / 2\right)\left\|B_{k}-F^{\prime}\left(x^{*}\right)\right\|_{M} \leqq & \left\|B_{k}-F^{\prime}\left(x^{*}\right)\right\|_{M}-\left\|B_{k+1}-F^{\prime}\left(x^{*}\right)\right\|_{M} \\
& +\sigma_{k}\left[\alpha_{3}\left\|B_{k}-F^{\prime}\left(x^{*}\right)\right\|_{M}+\alpha_{4}\right],
\end{aligned}
$$

and therefore summing both sides,

$$
(\alpha / 2) \sum_{k=1}^{\infty} \theta_{k}^{2}\left\|B_{k}-F^{\prime}\left(x^{*}\right)\right\|_{M}<+\infty .
$$

If some subsequence of $\left\{\left\|B_{k}-F^{\prime}\left(x^{*}\right)\right\|_{M}\right\}$ converges to zero, then (i) implies that the whole sequence converges to zero and thus (ii) is trivially satisfied. Otherwise, $\left\{\left\|B_{k}-F^{\prime}\left(x^{*}\right)\right\|_{M}\right\}$ is bounded away from zero, and the last equation implies that $\left\{\theta_{k}\right\}$ converges to zero. Statement (ii) follows.

Note that Theorem 3.4 holds regardless of how the sequence $\left\{x_{k}\right\}$ is generated provided (3.9) is satisfied. For example, suppose the sequence is generated by $x_{k+1}=$ $x_{k}-\lambda_{k} B_{k}{ }^{-1} F x_{k}$ where $\left\{\lambda_{k}\right\}$ is any sequence with $\left|\lambda_{k}-1\right| \leqq \lambda<1$ for $k \geqq 0$ and $\left\{B_{k}\right\}$ is generated by (3.5). Then, using the techniques of [2] and inequality (3.8), it is not difficult to show that if $F x^{*}=0$ then there is a constant $r \in(0,1)$ with

$$
\left\|x_{k+1}-x^{*}\right\| \leqq r\left\|x_{k}-x^{*}\right\|
$$

provided $\left(x_{0}, B_{0}\right)$ is close enough to $\left(x^{*}, F^{\prime}\left(x^{*}\right)\right)$. Hence, (3.9) is satisfied and if $\left\{\lambda_{k}\right\}$ converges to unity, (ii) above implies that

$$
\lim _{k \rightarrow+\infty}\left(\|\left[\lambda_{k}^{-1} B_{k}-F^{\prime}\left(x^{*}\right)\right] s_{k}|| /|| s_{k}||\right)=0 .
$$

Thus, Lemma 2.2 implies that $\left\{x_{k}\right\}$ is $Q$-superlinearly convergent. For $\lambda_{k} \equiv 1$, this reduces to a result of Broyden, Dennis, and Moré [2].

We now would like to show that Theorem 3.4 also yields the superlinear convergence of the version of the Davidon-Fletcher-Powell algorithm considered by Powell [8]. As mentioned at the beginning of this section, Powell chooses $\lambda_{k}$ via exact line minimization so that

$$
\nabla f\left(x_{k+1}\right)^{T} s_{k}=0 .
$$


THEOREM 3.5. Let $F: R^{n} \rightarrow R^{n}$ be differentiable in the open, convex set $D$ in $R^{n}$, and assume that for some $x^{*}$ in $D, F^{\prime}\left(x^{*}\right)$ is nonsingular, and $F^{\prime}$ is continuous at $x^{*}$. Let the sequence $\left\{x_{k}\right\}$ be defined by

$$
x_{k+1}=x_{k}-\lambda_{k} B_{k}^{-1} F x_{k},
$$

where the nonsingular matrix $B_{k}$ and scalar $\lambda_{k}$ are such that $F x_{k+1}{ }^{T} s_{k}=0$, and suppose that $\left\{x_{k}\right\}$ lies in $D$ and converges to $x^{*}$. If

$$
\lim _{k \rightarrow+\infty}\left\|\left[B_{k}-F^{\prime}\left(x^{*}\right)\right] s_{k}\right\| /\left\|s_{k}\right\|=0,
$$

then $\left\{\lambda_{k}\right\}$ converges to unity, $F x^{*}=0$ and $\left\{x_{k}\right\}$ converges $Q$-superlinearly to $x^{*}$.

Proof. Define, for $v, w \in R^{n}$,

$$
\langle v, w\rangle=\left[F^{\prime}\left(x^{*}\right)^{-1} v\right]^{T} w
$$

so that by the mean value theorem

$$
0=\left\langle F x_{k+1}, s_{k}\right\rangle=\left\langle F x_{k}, s_{k}\right\rangle+\left\langle F^{\prime}\left(x_{k}+t_{k} s_{k}\right) s_{k}, s_{k}\right\rangle
$$

where $\left|t_{k}\right|<1$. Hence,

$$
\left\langle F^{\prime}\left(x_{k}+t_{k} s_{k}\right) s_{k}, s_{k}\right\rangle=-\left\langle F x_{k}, s_{k}\right\rangle=\lambda_{k}^{-1}\left\langle B_{k} s_{k}, s_{k}\right\rangle .
$$

Therefore,

$$
\begin{aligned}
\left(\lambda_{k}-1\right)\left\langle F^{\prime}\left(x^{*}\right) s_{k}, s_{k}\right\rangle= & \lambda_{k}\left\langle\left[F^{\prime}\left(x^{*}\right)-F^{\prime}\left(x_{k}+t_{k} s_{k}\right)\right] s_{k}, s_{k}\right\rangle \\
& +\left\langle\left[B_{k}-F^{\prime}\left(x^{*}\right)\right] s_{k}, s_{k}\right\rangle .
\end{aligned}
$$

Using the definition of $\langle\cdot, \cdot\rangle$, the above equation can be written as

$$
\lambda_{k}-1=\lambda_{k} \alpha_{k}+\beta_{k}
$$

where

$$
\left|\alpha_{k}\right| \leqq\left\|F^{\prime}\left(x^{*}\right)-F^{\prime}\left(x_{k}+t_{k} s_{k}\right)\right\|\left\|F^{\prime}\left(x^{*}\right)^{-1}\right\|,
$$

and

$$
\left|\beta_{k}\right| \leqq \frac{\left\|\left[B_{k}-F^{\prime}\left(x^{*}\right)\right] s_{k}\right\|}{\left\|s_{k}\right\|} \cdot\left\|F^{\prime}\left(x^{*}\right)^{-1}\right\| .
$$

It follows that $\left\{\alpha_{k}\right\}$ and $\left\{\beta_{k}\right\}$ converge to zero, and thus (3.12) implies that $\left\{\lambda_{k}\right\}$ converges to unity as desired. The $Q$-superlinear convergence of $\left\{x_{k}\right\}$ to $x^{*}$ where $F X^{*}=0$ follows from Corollary 2.3.

Theorems 3.4 and 3.5 guarantee the $Q$-superlinear convergence of the DavidonFletcher-Powell method to a local minimum if $\lambda_{k}$ is chosen by an exact line search, and the sequence $\left\{x_{k}\right\}$ converges to $x^{*}$ in such a way that (3.9) is satisfied. For example, under the conditions on $F$ mentioned at the beginning of this section, Powell [8, Theorem 2] proved that there is a $\mu<1$ such that $\left\|x_{k}-x^{*}\right\| \leqq \eta \mu^{k}$ for some constant $\eta>0$ and all $k$. Hence, (3.9) holds, and we obtain an independent proof of Powell's result of $Q$-superlinear convergence.

Note, however, that $Q$-superlinear convergence to a zero of $F$ is also assured if $\left\{\lambda_{k}\right\}$ is any sequence which converges to unity and makes (3.9) hold. Therefore, a good choice of $\left\{\lambda_{k}\right\}$ would give us (3.9) while minimizing function evaluations; exact line minimization is an expensive way to do this. 
4. Extensions. The results of the previous section do not hold only for the Davidon-Fletcher-Powell method. In particular, the all-important (ii) of Theorem 3.4 holds for the following updates:

$$
\begin{aligned}
B_{k+1}= & B_{k}+\frac{\left(y_{k}-B_{k} s_{k}\right) s_{k}^{T}}{s_{k}^{T} s_{k}}, \\
B_{k+1}= & B_{k}+\frac{\left(y_{k}-B_{k} s_{k}\right) y_{k}^{T}}{y_{k}^{T} s_{k}}, \\
B_{k+1}= & B_{k}+\frac{\left(y_{k}-B_{k} s_{k}\right) s_{k}^{T}+s_{k}\left(y_{k}-B_{k} s_{k}\right)^{T}}{s_{k}^{T} s_{k}} \\
& -\frac{s_{k}^{T}\left(y_{k}-B_{k} s_{k}\right) s_{k} s_{k}^{T}}{\left(s_{k}^{T} s_{k}\right)^{2}} .
\end{aligned}
$$

Only in the third update is it necessary to take $B_{0}$ symmetric. To verify that these updates satisfy (ii) of Theorem 3.4, we only need to show that they satisfy a relationship like (3.8), and this is done in Lemmas 4.2 and 5.2 of [2]. The assumptions are as in Theorem 3.4, but for the first and third updates $M$ is the identity matrix, so that $F^{\prime}\left(x^{*}\right)$ need only be symmetric in the third update, while existence is all that is required in the first update. Conclusion (i) of Theorem 3.4 also holds but for the first and third updates it should be modified to read

$$
\lim _{k \rightarrow+\infty}\left\|B_{k}-F^{\prime}\left(x^{*}\right)\right\|_{F} \text { exists. }
$$

For more information on these methods and their properties see [2].

As we mentioned in the introduction, results on $Q$-superlinearity are usually proved by assuming $\left\{B_{k}\right\}$ converges to $F^{\prime}\left(x^{*}\right)$. Nothing, so far, in our results says that this approach cannot be applied to the quasi-Newton methods, but consider the following class of examples. Define $F: R^{n} \rightarrow R^{n}$ such that $f_{2}, \cdots, f_{n}$ are independent of $x_{1}$ and $f_{1}(x)=x_{1}$. Let $x_{0}$ have a zero in its first coordinate and $B_{0}$ zeros in its first row and column except for possibly the $(1,1)$ element. If $\left\{x_{k}\right\}$ and $\left\{B_{k}\right\}$ are generated by the Davidon-Fletcher-Powell or any of the above three methods, then the first row and column of $B_{k}$ will remain unchanged while the rest of $B_{k}$ will be the matrix generated by the corresponding method when applied to $f_{2}, \cdots, f_{n}$ as a function of the $n-1$ variables $\left(x_{2}, \cdots, x_{n}\right)$. In particular, the sequence $\left\{B_{k}\right\}$ does not converge to $F^{\prime}(x)$ for any $x$ in $R^{n}$. Of course, it is always possible for the iteration to terminate or to break down because of a division by zero, but by choosing $f_{2}, \cdots, f_{n}$ appropriately, convergence will be assured. Also note that the above example applies to the "damped" method

$$
x_{k+1}=x_{k}-\lambda_{k} B_{k}^{-1} F x_{k}, \quad \lambda_{k} \neq 0,
$$

and in fact to any quasi-Newton method such that $B_{k+1}$ is obtained from $B_{k}$ by adding a linear combination of matrices $p_{k} q_{k}{ }^{T}$ where $p_{k}$ and $q_{k}$ can be any of the vectors $s_{k}, B_{k} s_{k}, B_{k}{ }^{T} s_{k}, y_{k}, B_{k} y_{k}$, or $B_{k}{ }^{T} y_{k}$. In particular, this holds for the Huang class [5].

The first person to produce an example of this type was Powell [9]. He pointed out that if the Davidon-Fletcher-Powell method with exact line minimization is used to minimize the function $f\left(x_{1}, \cdots, x_{n}\right)=x_{1}{ }^{2}+g\left(x_{2}, \cdots, x_{n}\right)$, then the above behavior occurs from initial points of the type specified above. 
The previous results also apply to the so-called complementary or Fletcher-dual methods [3]. For example, the complementary Davidon-Fletcher-Powell method is given by

$$
x_{k+1}=x_{k}-H_{k} F x_{k}
$$

where

$$
\begin{aligned}
H_{k+1}= & H_{k}+\frac{\left(s_{k}-H_{k} y_{k}\right) s_{k}^{T}+s_{k}\left(s_{k}-H_{k} y_{k}\right)^{T}}{y_{k}^{T} s_{k}} \\
& -\frac{y_{k}^{T}\left(s_{k}-H_{k} y_{k}\right) s_{k} s_{k}^{T}}{\left(y_{k}^{T} s_{k}\right)^{2}} .
\end{aligned}
$$

The Fletcher-dual is therefore obtained by replacing $B$ 's by $H$ 's and interchanging $y_{k}$ and $s_{k}$. For this method, (i) and (ii) of Theorem 3.4 now read

(i) $\lim _{k \rightarrow+\infty}\left\|F^{\prime}\left(x^{*}\right)^{1 / 2} H_{k} F^{\prime}\left(x^{*}\right)^{1 / 2}-I\right\|_{p}$ exists,

(ii) $\lim _{k \rightarrow+\infty}\left(\|\left[H_{k}-F\left(x^{*}\right)^{-1} \mid y_{k}\|/\| y_{k} \|\right)=0\right.$,

where we are now assuming that $y_{k} \neq 0$ for $k \geqq 0$. Similar observations apply to the dual of the three methods defined in this section.

$Q$-superlinear convergence to a zero of $F$ also follows from (ii) above if we assume that $\left\{\left\|H_{k}^{-1}\right\|\right\}$ is uniformly bounded. In this case,

$$
\left[H_{k}-F^{\prime}\left(x^{*}\right)^{-1}\right] y_{k}=H_{k} F x_{k+1}-F^{\prime}\left(x^{*}\right)^{-1}\left[y_{k}-F^{\prime}\left(x^{*}\right) s_{k}\right],
$$

and, if $F$ satisfies the assumption of Theorem 2.2, then there is a $\gamma>0$ such that

$$
\left\|y_{k}\right\|=\left\|F x_{k+1}-F x_{k}\right\| \geqq \gamma\left\|x_{k+1}-x_{k}\right\| \text {. }
$$

All together,

$$
\lim _{k \rightarrow+\infty}\left(\left\|F x_{k+1}\right\| /\left\|x_{k+1}-x_{k}\right\|\right)=0 .
$$

This is just (2.5), so from (2.4) we see that $\left\{x_{k}\right\}$ must converge $Q$-superlinearly to $x^{*}$ and $F x^{*}=0$.

For the complementary Davidon-Fletcher-Powell method the matrices $B_{k}=H_{k}^{-1}$ are generated by

$$
B_{k+1}=B_{k}+y_{k} y_{k}^{T} / y_{k}^{T} s_{k}-B_{k} s_{k} s_{k}^{T} B_{k} / s_{k}^{T} B_{k} s_{k}
$$

In this case it is possible to prove, with the technique that Powell used in Theorem 3 of [8], that under the assumptions of Theorem 3.4 there is a positive integer $k_{0}$ such that, for any symmetric $B_{k_{0}} \in L\left(R^{n}\right)$, the sequences $\left\{s_{k}\right\},\left\{y_{k}\right\}$ and $\left\{B_{k}\right\}$ are well defined for $k \geqq k_{0}$ by (3.3) and (4.1), and, moreover, $\left\{\left\|B_{k}\right\|\right\}$ is uniformly bounded. Hence, the results of Section 3 apply to the complementary DavidonFletcher-Powell method without explicitly assuming that $\left\{\left\|H_{k}^{-1}\right\|\right\}$ is uniformly bounded.

5. Concluding Remarks. It is interesting to compare our results with those of McCormick and Ritter [6], and Ritter [10]. These authors prove, under more restrictive hypotheses than those of Theorem 2.2 , that $\left\{x_{k}\right\}$ is $Q$-superlinearly convergent if 


$$
\lim _{k \rightarrow+\infty} \frac{\left\|\left[B_{k}-F^{\prime}\left(x^{*}\right)\right]\left(x_{k+1}-x_{k}\right)\right\|}{\left\|F x_{k}\right\|}=0 .
$$

They fail to note that this condition is also sufficient (and hence, equivalent to (2.3)), and moreover, they do not prove that any of the single- or double-rank methods satisfy their hypotheses. They do make the interesting point that if

$$
\left\|\left[B_{k}-F^{\prime}\left(x^{*}\right)\right]\left(x_{k+1}-x_{k}\right)\right\| /\left\|F x_{k}\right\|=\Theta\left(\left\|F x_{k-\nu}\right\|\right)
$$

for some $\nu, 0 \leqq \nu \leqq n$, then

$$
\left\|x_{k+1}-x^{*}\right\| /\left\|x_{k}-x^{*}\right\|=\mathcal{O}\left(\left\|x_{k-\nu}-x^{*}\right\|\right) \text {. }
$$

In this case, it follows that the $R$-order of (2.2) is at least $r$ where $r$ is the unique positive root of $t^{\nu+1}-t^{\nu}-1=0$. See, for example, [7, p. 291]. It would be very interesting to prove that some quasi-Newton method satisfies (5.1) or some similar relationship that would guarantee an order greater than one.

We note that the techniques of this paper can also be used to establish $Q$-superlinear convergence of algorithms that use the quasi-Newton philosophy. For example, K. Brown and J. E. Dennis, in an unpublished work, have proven $Q$-superlinearity of the nonlinear least squares method given in [1].

Finally, readers familiar with the majorant approach to the convergence theory of nonlinear iterative methods will perhaps agree with us that (2.3) is a sort of directional-norm analogue of consistency, i.e., norm convergence of $\left\{B_{k}\right\}$ to $F^{\prime}\left(x^{*}\right)$. It would be interesting to find a reasonable sufficient condition for local convergence of (1.1) based on such a directional norm approach.

Acknowledgments. We would like to thank M. J. D. Powell for allowing us to publish the example mentioned in Section 4, P. T. Boggs who informed us of its existence, and C. G. Broyden, who taught us a lot of what we know about quasiNewton methods.

Department of Computer Science

Cornell University

Ithaca, New York 14850

1. K. M. Brown \& J. E. DenNis, JR., "A new algorithm for nonlinear least-squares curve fitting," in Mathematical Software, John R. Rice, Editor, Academic Press, New York, 1971, pp. 391-396.

2. C. G. Broyden, J. E. Dennis \& J. J. Moré, On the Local and Superlinear Convergence of Quasi-Newton Methods, Cornell Computer Science Technical Report 72-137, 1972; J. Inst. Math. Appl. (To appear.)

3. R. Fletcher, "A new approach to variable metric algorithms," Comput. J., v. 13, 1970 , pp. 317-322.

4. A. Goldstein \& J. Price, “An effective algorithm for minimization," Numer. Math., v. 10, 1967, pp. 184-189. MR 36 \#1084.

5. H. Y. HUANG, "Unified approach to quadratically convergent algorithms for function minimization." J. Optimization Theory Appl., v. 5, 1970, pp. 405-423. MR 44 \#6134.

6. G. MCCORMICK \& K. RitTER, "Methods of conjugate directions versus quasi-Newton methods," Math. Programming, v. 3, 1972, pp. 101-116. MR 46 \#1357.

7. J. M. ORTEGA \& W. C. RHEINBOLDT, Iterative Solution of Nonlinear Equations in Several Variables, Academic Press, New York, 1970. MR 42 \#8686.

8. M. J. D. POWELL, "On the convergence of the variable metric algorithm," J. Inst. Math. Appl., v. 7, 1971, pp. 21-36. MR 43 \#5698.

9. M. J. D. Powell, Private communication, 1972.

10. K. RITTER, "Superlinearly convergent methods for unconstrained minimization problems," Proc. ACM, Boston, 1972, pp. 1137-1145.

11. R. VoIGT, Rates of Convergence for Iterative Methods for Nonlinear Systems of Equations, Ph.D. Thesis, University of Maryland, College Park, Md., 1969. 